\title{
Research on the Operation Mode of "Wanghong" Store
}

\author{
Leng Fuchen \\ Wuhan Technology and Business University \\ Wuhan Hubei, 430065, China \\ 63831907@qq.com
}

\author{
Zhang Yanming* \\ Wuhan University of Communication \\ Wuhan Hubei, 430205, China \\ 342397941@qq.com \\ * Correspondence author
}

\begin{abstract}
With the development of Internet technology, "web celebrity" came into being, and has formed a unique economic development trend, namely "web celebrity Economy" which has developed rapidly in recent years. However, limitations like intensified competition, increased competition costs and fragile industrial chains keep cropping up. This paper analyzed the social factors of the network popular store formation and development of these stores, analyzed the operation mode mainly from the form of store ope ration and marketing strategy, and the limitations of the operation of the network popular store. Based on this, the paper put forward the operation strategies such as making original content, establishing trust with fans, focusing on after-sales service, improving store traffic, and optimizing store conversion, in order to provide reference for the development of online popular stores.
\end{abstract}

Keywords-wanghong economy; operation mode; industrial chain; restriction

\section{INTRODUCTION}

Since 2015, the topic "web celebrity" has been hotly debated, and search keywords such as "Wang Sicong(the only son of Wang Jianlin, China's richest man and the chairman of Wanda Group) Xueli(Wang's ex-girlfriend, a web celebrity)”, "Luo Zhixiang(Pop singer) Zhou Yangqing(Luo's girlfriend, a web celebrity)" and "Aaron Kwok Web Celebrity Girlfriend" frequently appear in the top ten of the Weibo hot search list. According to Taobao statistics, in the " $6 \cdot 18$ " promotion in 2017, among the top ten women's clothing stores, 7 were web stores operated by web celebrities. And the online popular store's transaction quantity was 2.5 times that of traditional online stores. In daily sales, the turnover of some new web popular stores exceeded 10 million Yuan a day, comparable to well-known clothing brands. In such a "web celebrity hot" environment, the business model of online popular stores has risen rapidly.

Online popular store is an economical industry model with bright prospects, attracting more and more individuals to participate in. From the initial personal operation, to the ecommerce stores and the professional wanghong companies, and then to various e-commerce platforms such as Jingdong, Meicheng.com and Taobao, these has confirmed the huge prospects of the wanghong economy. Therefore, the research on the operation mode of the wanghong store can guide the general network shop to win consumers' favor, know how to establish their own brand image, how to improve their brand value, how to properly operate and change so as to win a broader market space.

\section{WANGHONG STORE OVERVIEW}

\section{A. The annotation of the wanghong store}

"Wanghong" in Chinese also refers to the web celebrity. They are the one being followed by netizens because of an incident or some certain action in real life or on the internet, and can keep the popularity on the social platform [1]. In the early stage, Wanghong initially appeared on the mainstream media as models, and then cooperate with brands. Finally create their brands to earn money. Nowadays, wanghong attract a great amount of fans by their good-looking or character on the social media like Weibo and then introduce them to their own online stores to buy something. As these online stores boom, Wanghong economy and the business model of online popular stores emerge. The online popular stores are supported by wanghongs who attract fans by promotion or even sensationalization to earn from their popularities, which belongs to a business model of fan economy.

\section{B. Social factors in the formation of wanghong stores}

The formation of online stores, in addition to their own characteristics, is closely related to external factors. The influence of social factors is often the cause of the formation of nature, which constitutes the base for the development of wanghong economy.

\section{1) Economic factors}

With the development of the economy, there has emerged a diversified and enriched social culture that also drives the diversification of economy. The wanghong economy is one of the products of economic and cultural diversification. In recent years, with the stability of the online retail audience, the ecommerce market pattern has gradually stabilized. However, due to the limited development space in e-commerce market, competitions among e-commerce are increasingly intense. In this context, the business model of the online store supported by wanghong begins appearing. These online popular stores broke the competition mode of the existing e-commerce, and also became one of the important signs of e-commerce diversification. 


\section{2) Cultural factors}

It can be said that the wanghong economy is one of the fruits of differentiated development. Diversified culture results in diversification and differentiation of people's needs. People realize that they are different from others, and they also understand their own needs. The most important thing is that people's purchasing power has been greatly improved, and they can pay for their own ideas. Purchasing power stimulates cultural diversity, and cultural diversity leads to diversified demand, and demand diversification has given rise to a differentiated development of the sales model, which has given impetus to the popularity of the wanghong economy model [2].

\section{3) Individual factor}

The formation of the online famous shop is largely due to the people's conformity behavior. In general, people have no excessive denial of purchasing behavior. It can also be said that people are temporarily unable to make cognitive judgments on the purchase behavior itself, which provides the main premise for the expansion of herd mentality. In most cases, wanghong shops usually provide mainstream positive values which cater to the values of most people. Wanghong shop markets itself on the mainstream public opinion platform, for example, wanghongs making up on the live platform, publishing product soft text on Weibo. Take these methods to integrate product information into mainstream values, give hints to consumers and stimulate them to make purchases.

\section{WANGHONG STORE OPERATION OVERVIEW}

\section{A. Development status}

The evolution of wanghong has been in line with the development of the Internet. In 2003, the popularity of blogs promoted the appearance of a large number of grassroots network celebrities. Later in 2009, the popularity of Weibo and WeChat brought the e-commerce models. In 2015, there were a large number of wanghong on Kuaishou and other live streaming apps. The number of wanghong has risen rapidly with the development of major platforms, and the wanghong economy brought by it has been gradually in the right direction with unnegligible competitiveness.

Every online shop has its own labels and features, but no matter what the actual content is displayed, personal characteristics are indispensable. Being common cannot stand out; instead, one should become different from others to become unique. From the initial individual operation to the way to find a promoter, the current online store is facing both a bottleneck and an opportunity to move to a new height. The quality of products sold in the wanghong stores has become the most important concern, as it can affect the enthusiasm of the fans. Taking OnlyAnna as an example, she has about 1.68 million fans on Weibo. According to the customer, the product quality needs to be improved. Otherwise, it is difficult to strengthen the repurchase power.

\section{B. Interactive mode}

With the development of the wanghong economy, the face base has become more and more important, as it is the first purchasing power of wanghong stores. The fan base created with social media has played a critical role in the steady development of these online stores. Fan base is a group of likeminded people. Therefore, to build a fan base, you must have clear positioning, and maintain a good fan base, so as to maintain the existing purchasing power while stimulating potential purchasing power. Nowadays, with the popularity of various apps, video and live broadcasts are the best way to interact with fans and increase popularity. Interacting with your fan base before releasing a new product will have an unexpected effect on sales. Moreover, there are many ways to interact with fan base, such as increase the opportunities of exemption and send small gifts, display the matching items, which will be more noticeable than the single items, and initiate voting, question and answer, etc. In addition to interacting with the fan base, these methods can also collect data to analyze the trend of the store development and lay a good foundation for store operation.

\section{WANGHONG STORE OPERATION MODE}

\section{A. The operation mode of the wanghong store}

\section{1) Pseudo wanghong mode}

Instead of the fan base of the wanghong store, the pseudo wanghong store pays more attention to the quantity of their products sold, and has its own unique style to make consumers believe that these products are distinct from others. Although they aren't as powerful as the wanghong store, they have a certain sales performance. However, the fan base is not stable enough, and the influence is not strong enough. Therefore, it is necessary to rely on continuous launching new products to maintain the sales model.

\section{2) Wanghong mode}

Compared with the pseudo wanghong, wanghong pays more attention to the maintenance of their fixed fan base and the number of fans and affects the purchasing power and sales of fans through their popularity. They make a study of fans, styles, and operations with scientific theories, and adopt methods different from others. They mainly surpass competitors by highlighting their own characteristics to take a share in the corresponding market. The wanghong model has great secondary repurchase attractiveness through high popularity and good quality products, and can use big data to monitor data fluctuations in real time and analyze the data to realize store operation.

\section{3) Blockbuster mode}

Blockbuster mode is based on the traffic distribution rules of major platforms to create fashion trends, in line with the styles sought by consumers. It can achieve better traffic and rankings through customer preference, and increase sales.

\section{B. Marketing strategy of wanghong shop}

\section{1) Make poisoning}

If a brand wants to achieve sales goals through the wanghong, it should first of all shape the character of the wanghong. This kind of shaping involves a wide range of aspects, from the field to the individual's uniqueness. Whether these aspects can be appreciated by the customers? Whether they can bring wanghong the solid inherent fan base and 
potential customers? It is necessary to clear position the eyecatching point of the network celebrities, the type of product, as well as the target buyers.

\section{2) Output values in content}

When the wanghong is positioned in the early stage, they will gather their own fans, but they are not even the source of potential customers. So how to make these fans become purchasing power has become a new important issue. The hobbies and interests of the fans are wide, and vary swiftly with the development of the Internet, so it is very important to keep the fans unchanged. This requires the network celebrities to deliver some valuable content, including a good attitude towards life, a view of some events and things, and a unique but positive energy. The valuable topics made by the wanghong will promote interactions and get the fans' trust, thus enhancing their purchasing power [3].

\section{3) Focus on fan maintenance and product quality}

It's not hard to find that wanghong and fans are inextricably linked. Due to frequent interactions and fans' love for the wanghong, the wanghong are more like the mentor and friend of the fans. There is not only a relationship of interest, but also more trust, which paves the way for the following marketing. Therefore, maintaining a good fan base will bring a better fan economy. Wanghong need to enhance popularity, and attract more fans. Apart from the words and thoughts, wanghong should produce qualified products to win the stable clients. Moreover, these products should be loved by customers and have high quality and perfect after sales service to give customers a good purchasing experience so as to generate repurchase power.

Wanghong shop's high quality service is mainly reflected in the following aspects: (1) Wanghong's own personality shaping: form marketing scope with individuality according to the customers' hobbies; (2) Featured sales products: product quality goes first to win consumers' trust; (3) Price positioning: set the price for different customer groups, keep in line with product quality; (4) After-sales service: provide good aftersales service, promise to return the goods freely within the specified time; in case of quality problems, pay for public relations and let the customer group to supervise the sales of the store, provide quality protection; provide high quality guarantee.

\section{4) Strengthen self-personality promotion}

Generally, a wanghong has about hundreds of thousands or even millions of fans most of whom are loyal to their wanghong idol. So the weibo of the online store can gather great traffic. Stores should strengthen their individuality and know how to create a powerful impact on themselves or products. The network celebrities should spend a lot of time interacting with fans. In addition to sharing fanatic content with fans, they need to know how to properly implant business products and use their popularity to win traffic for sales. At the same time, in terms of delivery, it is a good way to establish a personalized image by creating the package with the wanghong's own characteristics, so that fans feel that both people and products are fully integrated into the elements they are looking for.

\section{5) Professional team operations}

Alongside the development of wanghong, a group of professional wanghong incubators have sprung up. They will arrange professional team to control character building and the final marketing and operation. Wanghong marketing, which used to be alone, is moving to a team mode. Professional big data analysis and cloud analysis will quickly and accurately grasp the data of the operation of the network celebrities' stores The professional team adjusts the operation mode according to these effective data in a timely manner. Fully integrate the trend and the preferences of senior fans, which not only reduce the difficulty of selection for consumers, but also greatly increase traffic, exposure and earnings.

\section{Restrictions of the operation of net red stores}

\section{1) Deeper competition}

The wanghong economy has become popular in recent years, and a large amount of capital has flown into this market. The wanghong shop was originally the fruit of the differentiated sales model, but due to the inflow of a large amount of capital, the online stores with the similar sales mode of the wanghong store keep cropping up, which increases the degree of homogenization. If there is no other obvious competition for traffi, it is likely to cause vicious competition, leading to the collapse and reconstruction of such store ecosystems [4]. As far as the current market ecology is concerned, competition is heating up, and the vicious circle is beginning to emerge.

\section{2) Higher competitive costs}

The competitive cost here is not only the cost of the commodity itself, but the cost of the package. For the wanghong store, the most important thing is network celebrities' attendance fee and promotion fee. According to statistics, the attendance fee of network stars increased by nearly $26.7 \%$ compared with last year, which is closely related to the development of network culture. The exposure rate of web celebrity stars is the cornerstone of web celebrity shops. Without network traffic, it is impossible to create successful web celebrity shops. Therefore, the investment in web celebrity star exposure rate is always made regardless of cost. The truth is that the investment is often beyond the return.

"We media" is one of the important publicity channels for web celebrity stores. However, behind each successful "we media” are a large amount of capital investment. The market has been dominated by those successful wanghong shops. New shops don't have such publicity channels, and that means tremendous pressure and low survival rate. Therefore they are not strong enough to shock the industry or even break the existing or potential vicious cycle.

\section{3) Vulnerable industrial chain}

The wanghong economy is more dependent on personal traffic, and the quality reputation of the product itself is publicized based on the recommendation of a certain wanghong. Given this, if the wanghong has a public relations crisis, even if it is not related to the goods, it will seriously affect the normal operation of the store. Behind the wanghong, there is usually a series of profit chains which can be damaged by taking Wanghong as the breaking point. In a word, 
compared with the traditional economy, the wanghong sales model is relatively fragile.

\section{Operation STRATEGY OF WANGHONG STORE}

\section{A. Business strategy}

\section{1) Output content and values}

With the emergence of the wanghong economy from the very beginning to the current saturation, the fans' demands for the wanghongs are getting higher and higher, so the content shared by the wanghongs is full of variety. Wanghongs need to share what the fans like. Apart from that, the cost performance of the products is an important development path for the wanghong economy. The wanghong need to understand the needs of the fan groups and grasp their interests, and add unique ideas, so that the output can increase the value, making fans become potential customers [4].

\section{2) Original content and platform}

With the rapid development of the Internet, what fans are interested in varies rapidly. And various social platforms provide fans with a wide range of contentc, so original content will be particularly important. The original content can not only improve the traffic, but also increase the fans appreciation for the wanghong's taste [5]. It is also important to choose a certain platform as the development base, and use other platforms to assist in the promotion. Make original content to reduce the aesthetic fatigue of the fan.

\section{3) Building trust and promote sales}

When the fans become the die-hard fans by being attracted by the personality of the network celebrity, the celebrity build fans' trust in the celebrity himself and products, and make them willing to buy through various aspects of the display. Creating a sense of trust is the key to facilitating the potential customer to pay, which requires the wanghong to know the hobbies of the fan base. Sales promotion is based on trust. When fans have a desire to purchase products launched by the wanghong, but hesitate, wanghong needs to find ways to promote their transformation from "intend to deal" to "intend to trade now".

\section{4) Emotional investment and after-sales maintenance}

The emotional investment takes place after the first purchase. It is a process in which a wanghong induces customers to repurchase through emotional marketing, increasing purchasing power and the stickiness of the wanghong. After-sales maintenance is based on the emotional investment. Wanghong will offer intimate after-sales service for the products sold, in terms of service and quality, to increase the repurchasing power of fans.

\section{B. Management strategy}

\section{1) Guarantee store traffic}

Wanghong must understand their store traffic, grasp the promotion ratio of various channels, and analyze the structure of store traffic, maintain and enhance the fan effect, offer effective data to promoters, and use the team to make scientific and reasonable adjustment.

\section{2) Optimize store experience}

One of the important factors that the wanghong's store is eye-catching to the fan community is wanghong's own individuality. The web celebrities need to optimize the shop interface with unique style, enhance the adjustment of visual and artistic aspects, collect and analyze the data, and optimize the evaluation.

\section{3) Make use of store data}

In the era of big data, data is the most convincing evidence to explain everything. Data analysis can get accurate and effective information in the first place. Therefore, wanghong and the team of store should cultivate the habit of drawing the daily forms of the store, and make the form with the data of the store promotion. Regularly analyze the activity and the plan, and summarize the sales condition and efficiently collect and analyze the store data.

\section{CONCLUSION}

The wanghong store model is a new business breakthrough. As netizens are gradually picking up rationality, the development of wanghong stores faces great uncertainty. Excellent wanghong sellers and various network incubators, with the advantage of supply chain end product manufacturers, can bridging perfectly to empower producers better bargaining capability during negotiations. However, with the gradual expansion of network celebrities and the expansion of supply chain demand, it is increasingly difficult for wanghong stores to meet the requirements of supply chain response speed. Therefore, the wanghong economy will finally back to the internal cost of the product, returning to the integration of the supply chain and the traditional construction.

\section{REFERENCES}

[1] Chen Rongrong. Prosperity and Mystery: Wanghong and Wanghong Economy [J].Business.2016(28)

[2] Cao Xiaofang. The Development of Business of Web Celebrities under the Fan Economy[J].Business.2016(23)

[3] Yuan Dong. Wanghong Economy: From Overseas Pioneer, Turning Fans into Cash is the Core Model [N].2016-04-05 (011)

[4] Xiao Zanjun, Zhang Hui.Calm Thoughts on the Web Celebrity Economy Boom [J].China Newspaper Industry.2016(17)

[5] Wang Xiaoying. Wanghong Economy Activates Fan Purchasing Power; Brand Innovation Creates New Entrepreneurship [N]. Communication Information News.2015-09-09 (A1) 